\title{
Performance characteristics of lipoprotein-associated phospholipase A2 activity assay on the Dimension Vista analyser and preliminary study of a healthy Italian population
}

\author{
Alessandro De Stefano, Liliana Mannucci*, Renato Massoud, Sergio Bernardini, Claudio Cortese \\ Department of Experimental Medicine and Surgery, University of Rome Tor Vergata, Rome, Italy \\ *Corresponding author: lilianamannucci@gmail.com
}

\begin{abstract}
Introduction: Lipoprotein-associated phospholipase A2 (Lp-PLA $)$ is a marker of vascular inflammation associated with coronary heart disease and stroke. We evaluated analytical performance of the PLAC ${ }^{\circledR}$ Activity Test on Siemens Dimension Vista ${ }^{\circledR} 1500$ analyzer and measured Lp-PLA activity in Italian adults to establish reference intervals (Rls) and evaluate correlation with circulating lipids and age.
\end{abstract}

Materials and methods: The evaluation protocol consisted of precision, linearity, sensitivity, method comparison, substrate depletion ("hook") effect and interference assessment. Inhibitor (Darapladib) effect was also evaluated. Lp-PLA 2 activity was measured in 250 healthy donors (123 males, 127 females, aged $18-70$ years). Central $95 \%$ Rls were established using nonparametric statistics.

Results: Intra-assay and inter-assay precision showed CVs of $0.6 \%-1.4 \%$ and $0.9 \%-2.0 \%$, respectively. Linearity replicates showed $R^{2}>0.98$. Limit of quantitation was $5.8 \mathrm{U} / \mathrm{L}(\mathrm{CV}=9.4 \%)$. Bland Altman plot showed bias $-0.9,95 \%$ limits of agreement -6.5 - 4.72. Passing-Bablok regression showed excellent correlation (Slope $=1.02,95 \% \mathrm{Cl}: 1.01$ to 1.03; Intercept $=-1.86,95 \% \mathrm{Cl}$ : 3.08 to $-1.26 ; \mathrm{R}^{2}=0.999$ ). No "hook effect" was observed at Lp-PLA activities $\leq 1000 \mathrm{U} / \mathrm{L}$. Average Lp-PLA 2 activity in 250 healthy donors was $182 \pm 44 \mathrm{U} / \mathrm{L}$ (mean \pm SD). Males showed statistically significant higher activities than females $(P<0.001)$. Rls were $107-265 \mathrm{U} / \mathrm{L}$ for males and $84-225 \mathrm{U} / \mathrm{L}$ for females. Moderate significant correlation

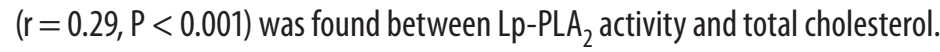

Conclusions: The PLAC ${ }^{\oplus}$ Activity Test shows very good performance characteristics on Dimension Vista ${ }^{\circledR} 1500$.

Key words: Lp-PLA 2 ; assay validation; cardiovascular disease; Darapladib; reference intervals

\section{Introduction}

Lipoprotein-associated phospholipase A2 (Lp$\mathrm{PLA}_{2}$ ), also known as platelet-activating factor acetyl-hydrolase, is a calcium-independent serine lipase which was initially identified by its hydrolysing action on the acetyl group at the sn-2 position of platelet-activating factor (1). It is now known that Lp-PLA $A_{2}$ can hydrolyse a broad spectrum of substrates, including oxidized and polar phosphatidylcholines (2). Lp-PLA 2 is synthesized by macrophages and other inflammatory cells and circulates in human blood mainly bound to lowdensity lipoprotein (LDL) particles (80 - 85\%) and, to a lesser extent, to high-density lipoprotein
(HDL) (3). Although it is considered an atheroprotective enzyme, the hydrolysis of oxidized phospholipids generates lysophospholipids, compounds that have a pro-inflammatory function (4). Several lines of evidence indicate that oxidized LDL play a critical role in atherogenesis and Lp$\mathrm{PLA}_{2}$ has been shown to be involved in the oxidative modification of LDL in the vascular wall by hydrolysing oxidized phospholipids, generating lysophosphatidylcholine and oxidized non-esterified fatty acids, which are strong pro-inflammatory mediators contributing to the formation of the atherosclerotic plaque (4-6). Lp-PLA 2 is a highly 
specific marker of vascular inflammation and its role in the development and progression of atherosclerosis has been suggested by various authors (6-8). High Lp-PLA 2 activity during atherosclerotic plaque formation and other inflammatory processes have been shown in several studies and there is increasing evidence that this enzyme is present within atherosclerotic lesions and enriched in vulnerable regions (6,8-11). Along with the well-known inflammatory marker high sensitivity C-reactive protein, $\mathrm{Lp}-\mathrm{PLA}_{2}$ has gained increasing significance and has been identified as an independent predictor of cardiovascular disease (CVD) in several studies, with elevated Lp-PLA 2 activities associated with an increased risk of CVD $(7,8,12,13)$. Moreover, since half of first cardiovascular events occur in apparently healthy subjects showing normal lipid profiles, Lp-PLA 2 measurement can be of particular clinical relevance in primary prevention, as it could help identify patients with unrecognized cardiovascular risk when the evaluation of traditional risk markers is not sufficiently informative $(8,14,15)$. A consensus panel issued in 2008 recommended testing Lp-PLA 2 not only as a diagnostic test for vascular inflammation but also in adjunct to traditional risk factor assessment in individuals with moderate or high risk of cardiovascular disease as defined by Framingham risk scores (16). This would allow improved identification of patients at high or very high CVD risk who would benefit from intensification of lipidmodifying therapy, and a lower LDL-cholesterol goal concentration is suggested when Lp-PLA 2 activities are high (17). Different assays for the measurement of Lp-PLA 2 activity in human plasma and serum have been developed but to date only the PLAC ${ }^{\circledast}$ Test for Lp-PLA 2 Activity Kit (diaDexus, Inc., San Francisco, USA) has received clearance by the Food and Drug Administration (FDA), in December of 2014.

In the present study we evaluated the analytical characteristics of the PLAC ${ }^{\oplus}$ Test on a Siemens Dimension Vista 1500 analyzer (Siemens Healthcare Diagnostics Inc., Newark, USA), which is currently installed in our Core Clinical Chemistry Laboratory in an automated configuration. Since reference in- tervals (RIs) for Lp-PLA 2 activity in the Italian population have not been reported yet, we analysed serum samples from a cohort of Italian healthy volunteers and evaluated the distribution of $L p-P L A_{2}$ activity and the correlation of serum Lp-PLA $A_{2} a c-$ tivity with circulating lipids and age.

\section{Materials and methods}

\section{Subjects}

For the reference population, serum samples were retrospectively selected from stored spare samples obtained from healthy volunteers, which were previously recruited at Tor Vergata University Hospital. We selected spare serum samples from 250 apparently healthy volunteers (123 males, 127 females), median age 37 (18 - 70) years. All subjects had undergone a visit consisting of a medical history, a physical examination, blood pressure measurement, and routine laboratory blood tests (clinical chemistry, haematology, coagulation), and had given informed consent for the collection, storage and/or reuse of blood samples. Exclusion criteria for our study were history and/or signs of CVD, any chronic disease or severe medical condition, alcohol and/or drug abuse, hypertension (systolic blood pressure $\geq 140 \mathrm{~mm} \mathrm{Hg}$ and/or diastolic blood pressure $\geq 90 \mathrm{mmHg}$ or under antihypertensive medication), total cholesterol $>5.2$ $\mathrm{mmol} / \mathrm{L}$, triglycerides $>1.8 \mathrm{mmol} / \mathrm{L}$, and use of lipid-lowering agents. Fasting blood samples had been collected into BD Vacutainer ${ }^{\circ} \mathrm{SST}^{\mathrm{TM}}$ ॥ $\mathrm{Ad}$ vance Plus serum separator tubes (Becton, Dickinson and Company, Franklin Lanes, USA) following standard procedures. Sample storage conditions were derived from previously published stability studies $(18,19)$. Briefly, samples were selected on a daily basis after completion of routine laboratory assessments, which were performed within 1 - 2 hours of collection. Selected blood samples were anonymized and either stored at $4{ }^{\circ} \mathrm{C}$ and analysed for Lp-PLA 2 activity within 1 week or aliquoted and stored at $-70{ }^{\circ} \mathrm{C}$ for a maximum of 3 months and then analysed after a single freezethaw cycle. 


\section{Methods}

$L p-P L A_{2}$ enzyme activity

Lp-PLA $A_{2}$ activity was determined on an open, user-defined channel on the Siemens Dimension Vista 1500 automated chemical analyser (Siemens Healthcare Diagnostics Inc., Newark, DE, USA) using the $\mathrm{PLAC}^{\circ}$ Test Activity Kit developed by diaDexus (diaDexus, Inc., San Francisco, CA) for the quantitative determination of $\mathrm{Lp}-\mathrm{PLA} \mathrm{A}_{2}$ activity in human plasma and serum. Principle of the test is based on Lp-PLA ${ }_{2}$ hydrolysing the $s n-2$ position of the substrate, 1-myristoyl-2-(4-nitrophenylsuccinyl) phosphatidylcholine, and producing a coloured reaction product, 4-nitrophenol. Lp-PLA 2 activity was determined by spectrophotometrically monitoring the rate of 4-nitrophenol formation at $405 \mathrm{~nm}$ (secondary wavelength $510 \mathrm{~nm}$ ) for 10 minutes, according to previously published methods $(20,21)$. A minimum serum sample volume of $200 \mu \mathrm{L}$ was required. Total reagent volume to fill an empty Siemens Flex (Siemens Healthcare Diagnostics Inc., Newark, USA) cartridge was $12.0 \mathrm{~mL}$ for R1 (buffer) and $3.2 \mathrm{~mL}$ for R2 (substrate). Working volumes for a single test were $100 \mu \mathrm{L}$ for R1 and $25 \mu \mathrm{L}$ for R2. Under these conditions, 104 tests could be run with each cartridge. A 5-point calibration was performed using a set of five calibrators made with purified recombinant Lp-PLA 2 protein to generate activities of $0,50,100,250$, and $400 \mathrm{U} / \mathrm{L}$, respectively. Calibrators were measured in triplicate. Calibration was verified by measuring high and low activity controls provided with the kit. Lp-PLA, activity was derived from the calibration curve by plotting change in absorbance versus Lp-PLA 2 activity in U/L.

Serum samples for method validation were provided by diaDexus. These samples had been previously obtained from healthy volunteers and stored at $-70^{\circ} \mathrm{C}$. For the present study, samples were shipped on dry ice and were received frozen.

\section{Precision}

Assay precision was evaluated according to the EP15-A2 document of the Clinical and Laboratory Standards Institute (CLSI), using 5 different samples ( 2 controls and 3 serum samples) with known
Lp-PLA 2 activity distributed throughout the calibration range of the assay (22). Intra-assay precision was evaluated by running 20 replicates of each sample in a single assay run. Inter-assay precision was evaluated by running 4 replicates of each sample on 5 different days, with a new calibration every day.

\section{Sensitivity}

Assay sensitivity was evaluated by analysing 4 samples with known low Lp-PLA 2 activity (range 0 - $10 \mathrm{U} / \mathrm{L}$ ) in 5 replicates over 5 calibration runs on 5 different days, for a total of 25 replicates for each sample. As specified by the manufacturer, predefined goals for bias and imprecision were respectively $\pm 5 \%$ difference from reference value and a coefficient of variation (CV) $<20 \%$ at the limit of quantitation (LoQ).

\section{Linearity}

Assay linearity was determined by serial recovery studies performed on 3 pairs of different serum samples with high or low Lp-PLA 2 activity (range: 96 $328 \mathrm{U} / \mathrm{L})$. In each serum pair, samples were mixed at ratios of 0:100, 10:90, 20:80, 30:70, 40:60, 50:50,60:40, 70:30, 80:20, 90:10, and 100:0, for a total of 11 activity levels which were measured in duplicate and then compared to expected calculated results.

\section{Method comparison}

Since there is no gold standard for Lp-PLA 2 activity to assess assay accuracy, the Bland-Altman plot was used to perform method comparison between the Dimension Vista 1500 and the analytical platform used by the manufacturer. Lp-PLA 2 activity was measured in duplicates in a single assay run on 40 blind serum samples spanning an activity range of $5-365 \mathrm{U} / \mathrm{L}$, which were provided by diaDexus. Our results were then compared to the activity values previously measured by diaDexus on the same samples using a Beckman Coulter $\mathrm{AU} 400^{\circ}$ analyser (Beckman Coulter Inc., Brea, USA).

\section{Hook effect}

The effect of substrate depletion due to high concentrations of Lp-PLA 2 protein on the measure- 
ment of its enzymatic activity ("hook effect") was evaluated as follows: purified recombinant Lp$\mathrm{PLA}_{2}$ was diluted into the calibrator matrix to nominal Lp-PLA 2 activities of $0,50,75,150,225,300$, $400,600,750,1000,1250,1500$, and $2000 \mathrm{U} / \mathrm{L}$. These samples were then analysed in duplicate. The concentration of recombinant Lp-PLA 2 was measured by the Bradford protein assay.

\section{Analytical interferences}

For interference testing, selected serum samples with Lp-PLA 2 activity in the range of 96 to $239 \mathrm{U} / \mathrm{L}$ were spiked with different levels of potentially interfering endogenous substances. A 6841.5 $\mu \mathrm{mol} / \mathrm{L}$ bilirubin stock solution (Sigma-Aldrich Inc, St. Louis, MO, USA) was diluted in $0.1 \mathrm{M} \mathrm{NaOH}$ and spiked in serum samples to a concentration of $342.1 \mu \mathrm{mol} / \mathrm{L}$. A $200 \mathrm{~g} / \mathrm{L}$ albumin stock solution (Calbiochem, San Diego, CA, USA) was diluted in distilled water and spiked in serum samples to a concentration of $50 \mathrm{~g} / \mathrm{L}$. Baseline albumin concentration was also measured in each sample and total albumin (baseline + supplementary) was taken into account for interference evaluation. Haemolysate solution (in-house, $105 \mathrm{~g} / \mathrm{L}$ haemoglobin) and Intralipid $20 \%$ emulsion (Sigma-Aldrich Inc, St. Louis, USA; $20 \%=2.3 \mathrm{mmol} / \mathrm{L}$ triglycerides) were ready to use. Serum samples with respectively $1.5 \mathrm{~g} / \mathrm{L}, 1.25 \mathrm{~g} / \mathrm{L}$, and $1.0 \mathrm{~g} / \mathrm{L}$ haemolysate were prepared. Intralipid ${ }^{\oplus}$ emulsion to a final triglycerides concentration of $2.8 \mathrm{mmol} / \mathrm{L}$ was added to selected serum samples. For each substance, controls were prepared by spiking serum samples with the corresponding solvent, when appropriate. Bilirubin, albumin and Intralip$\mathrm{id}^{\circledR}$ (triglycerides) were measured on the Dimension Vista 1500 instrument. Haemoglobin (haemolysate) stock solution was measured on the XE-2100 automated haematology analyser (Sysmex, Kobe, Japan).

\section{Lp-PLA $A_{2}$ enzyme inhibition}

A $5 \mathrm{mM}$ stock solution of the Lp-PLA $A_{2}$ inhibitor Darapladib was serially diluted in dimethyl sulfoxide (DMSO) in order to obtain 11 dilution levels with inhibitor concentrations ranging from 0.50 $\mathrm{nM}$ to $500 \mathrm{nM}$. Four different serum samples (Lp-
PLA 2 activity range: 97 - $243 \mathrm{U} / \mathrm{L}$ ) were divided into $232 \mu \mathrm{L}$ aliquots and $8 \mu \mathrm{L}$ of each Darapladib dilution were spiked into individual aliquots from all 4 sera, for a total of 44 test samples. Lp-PLA 2 activity was measured in inhibitor spiked aliquots, unspiked aliquots, and aliquots spiked with $8 \mu \mathrm{L}$ of DMSO alone as a control. Measured values were used to fit a dose-response curve for the determination of the in vitro half-maximal inhibitory concentration (IC50) of Darapladib.

\section{On-board reagent stability}

The R1 (buffer) and R2 (substrate) reagents were transferred to the specific Flex cartridge and stored at $2-8^{\circ} \mathrm{C}$ in the refrigerated compartment of the Dimension Vista 1500 instrument for a maximum of 4 weeks. Lp-PLA 2 activity was measured in triplicate on high and low controls 3 times a week over a window of 28 days, for a total of 72 determinations, always using the same reagent set and calibration curve performed at day 0. Lp-PLA 2 activity variations of $\pm 5 \%$ from initial value were considered acceptable.

\section{Statistical analysis}

Statistical analysis was performed using MedCalc for Windows, version 13.0 (MedCalc Software, Ostend, Belgium). Method comparison between the Dimension Vista 1500 and the Beckman AU400 was performed by Bland-Altman plot and PassingBablok regression analysis. The KolmogorovSmirnov test was used to assess the normality of distribution of Lp-PLA 2 activity in the whole study population, and in males and females separately. Reference intervals were defined according to CLSI document C28-A3 using the nonparametric method (minimum sample size required for each partition group: $\mathrm{N}=120$ ) and central $95^{\text {th }}$ percentile of reference values (23). Differences between genders were tested by unpaired t-test. Spearman's and Pearson's correlation test was used to test the association between serum Lp-PLA 2 activity and serum concentrations of total cholesterol, LDL-cholesterol (directly measured), HDL-cholesterol, and triglycerides and the association between serum Lp-PLA 2 activity and age in the study population. The values $\mathrm{P}<0.05$ were considered statistically significant. 


\section{Results}

Intra-assay variability analysis yielded within-run CVs ranging from $0.6 \%$ to $1.4 \%$ (Table 1 ). Inter-assay variability analysis yielded between-run CVs ranging from $0.9 \%$ to $2.0 \%$ (Table 1 ).

LoQ was determined as $5.8 \mathrm{U} / \mathrm{L}$ with a CV of $9.4 \%$. Linear regression analysis of Lp-PLA $A_{2}$ activity values (range: 96 - $328 \mathrm{U} / \mathrm{L}$ ) from the serial recovery assay samples compared to expected activity values resulted in slopes ranging from 1.009 to 1.013 , intercepts ranging from - 1.134 to 3.097 and $R^{2}$ ranging from 0.998 to 0.999 . Detailed results are reported in Table 2.

The Bland-Altman plot used for the evaluation of agreement between the Dimension Vista ${ }^{\circledR} 1500$ and the Beckman AU400 (Figure 1A) showed a mean difference of - $0.9 \mathrm{U} / \mathrm{L}(95 \% \mathrm{Cl}$ - 1.82 to 0.02$)$, lower limit of $-6.5 \mathrm{U} / \mathrm{L}(95 \% \mathrm{Cl}$ - 8.10 to - 4.94), upper limit of $4.7 \mathrm{U} / \mathrm{L}$ (95\% Cl: 3.14 to 6.30). Results of Passing-Bablok regression analysis are also shown (Figure 1B) (Slope $=1.02,95 \% \mathrm{Cl}: 1.01$ to 1.03; Intercept $=-1.86,95 \% \mathrm{Cl}:-3.08$ to $-1.26 ; \mathrm{R}^{2}=0.999$ ).

No "hook effect" was observed for measured absorbance up to $450 \mathrm{OD} \times 10^{3} / \mathrm{min}$, corresponding to a nominal recombinant Lp-PLA 2 activity of 1000 U/L (Figure 2). Recovery was within 90 - 110\%.

Albumin, bilirubin, haemoglobin, and triglycerides $\left(20 \%\right.$ Intralipid $^{\circledR}$ ) were tested as potentially interfering endogenous substances as described in the Methods section. No interference was observed for haemoglobin up to $1.5 \mathrm{~g} / \mathrm{L}$, bilirubin up to 342

TABLE 1. Precision evaluation of the lipoprotein-associated phospholipase A2 activity assay on the Dimension Vista analyzer

\begin{tabular}{|c|c|c|c|c|c|c|}
\hline \multicolumn{4}{|c|}{ Intra-assay imprecision } & \multicolumn{3}{|c|}{ Inter-assay imprecision } \\
\hline Study sample & Lp-PLA ${ }^{\prime}, U / L$ & $\% \mathrm{CV}$ & $\begin{array}{c}\text { Package } \\
\text { insert } \% \mathrm{CV}\end{array}$ & Lp-PLA ${ }_{2}, U / L$ & $\% \mathrm{CV}$ & $\begin{array}{c}\text { Package } \\
\text { insert \%CV }\end{array}$ \\
\hline Low Control & $121(1.5)$ & 1.2 & - & $121(2.4)$ & 2.0 & - \\
\hline High Control & $295(2.5)$ & 0.8 & - & $301(4.1)$ & 1.4 & - \\
\hline Serum \#1 & $98(0.6)$ & 0.6 & - & $99(0.9)$ & 0.9 & - \\
\hline Serum \#2 & $200(2.9)$ & 1.4 & - & $201(2.8)$ & 1.4 & - \\
\hline Serum \#3 & $254(2.4)$ & 0.9 & - & $258(3.0)$ & 1.2 & - \\
\hline Range & $98-295(0.6-2.9)$ & $0.6-1.4$ & $1.3-1.4$ & $99-301(0.9-4.1)$ & $0.9-2.0$ & $1.9-2.6$ \\
\hline
\end{tabular}

Lp-PLA 2 - lipoprotein-associated phospholipase A2. Lp-PLA 2 activities are presented as mean (standard deviation). CV - coefficient of variation. For intra-assay imprecision evaluation, 20 replicates of each sample were measured in a single assay run. For inter-assay imprecision evaluation, 4 replicates of each sample were measured on 5 different days. Ranges of intra- and inter-assay \%CVs obtained by the manufacturer on a Beckman Coulter AU400 analyzer (package insert) are also shown.

TABLE 2. Linearity evaluation of the lipoprotein-associated phospholipase A2 activity assay on the Dimension Vista analyzer

\begin{tabular}{|c|c|c|c|c|c|c|}
\hline \multirow{2}{*}{ Sample pair } & \multirow{2}{*}{$\begin{array}{c}\text { High activity } \\
\text { sample, U/L }\end{array}$} & \multirow{2}{*}{$\begin{array}{l}\text { Low activity } \\
\text { sample, U/L }\end{array}$} & \multicolumn{3}{|c|}{ Linear regression } & \multirow{2}{*}{ Package insert } \\
\hline & & & Slope & Intercept & $\mathbf{R}^{2}$ & \\
\hline$\# 1$ & 299 & 96 & 1.01 & -1.13 & 0.999 & Slope: 0.98 - 1.03 \\
\hline$\# 2$ & 316 & 97 & 1.01 & -0.17 & 0.999 & Intercept: - 6.8 - 10.7 \\
\hline$\# 3$ & 328 & 97 & 1.01 & 3.10 & 0.998 & $R^{2}: 0.995-0.999$ \\
\hline
\end{tabular}

Three pairs of serum samples with high and low Lp-PLA 2 activities were used to obtain $11 \mathrm{Lp}-\mathrm{PLA}$ activity levels in a serial recovery assay. LP-PLA 2 activity was measured in duplicate in each level and mean values were compared to expected activity values. Linearity results obtained by the manufacturer on a Beckman Coulter AU400 analyzer (package insert) are also shown. 
A

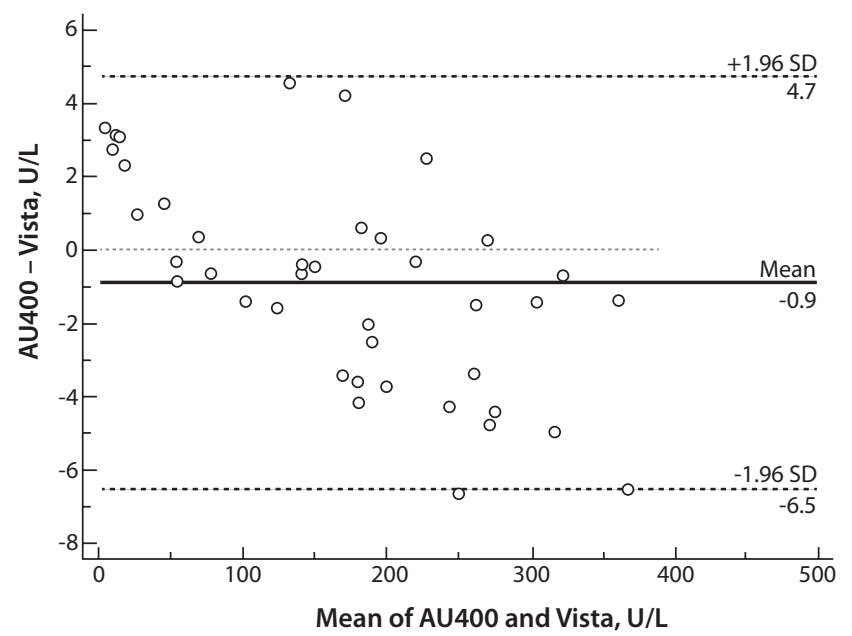

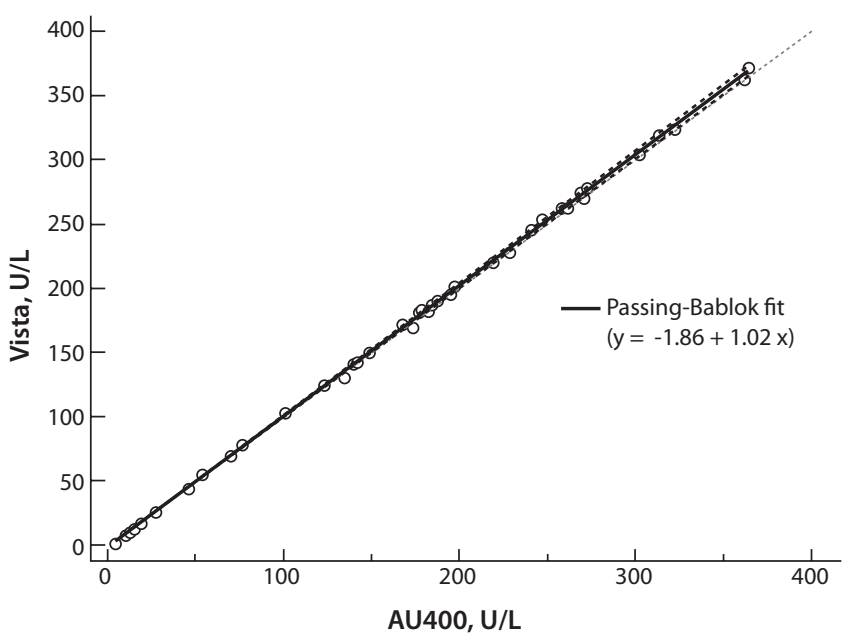

FIGURE 1. Comparison of lipoprotein-associated phospholipase $A 2\left(L p-P L A_{2}\right)$ activity values $(N=40)$ obtained on VISTA and AU400. (A) In the Bland-Altman plot the solid line shows mean difference, while the dashed lines show the \pm 1.96 SD. (B) In the PassingBablok regression analysis, the dashed lines show the $95 \%$ confidence interval $(\mathrm{Cl})$, while dotted line represents identity $(\mathrm{X}=\mathrm{Y})$.

$\mu \mathrm{mol} / \mathrm{L}$, albumin up to $90 \mathrm{~g} / \mathrm{L}(50 \mathrm{~g} / \mathrm{L}$ spike +40 $\mathrm{g} / \mathrm{L}$ in serum), and triglycerides up to $2.8 \mathrm{mmol} / \mathrm{L}$.

Dose-response data for the determination of the in vitro IC50 of Darapladib were obtained by high precision Lp-PLA 2 activity inhibition assay $(0.50$ to $500 \mathrm{nM}$ Darapladib spiked into multiple serum aliquots, baseline Lp-PLA 2 activity range: $97-243$ $\mathrm{U} / \mathrm{L}$ ). These data were used to fit a dose-response

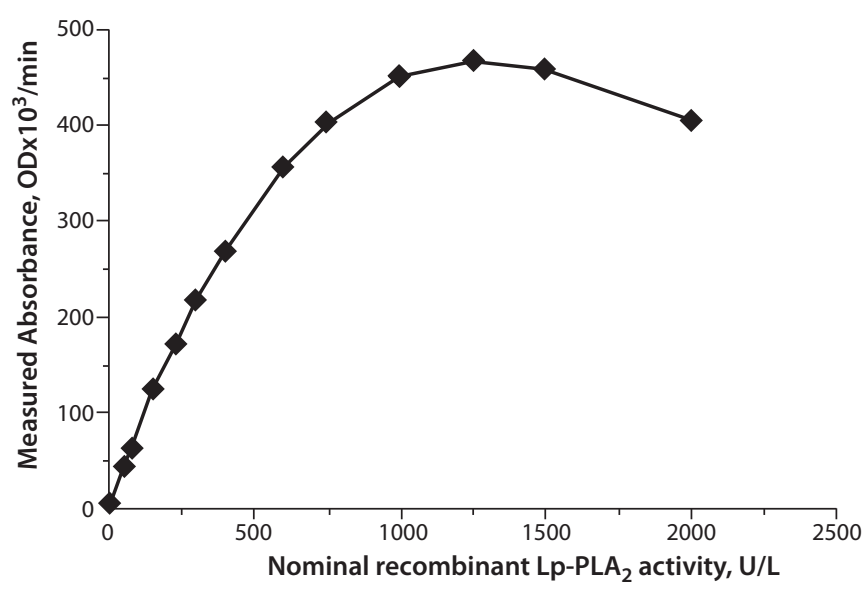

FIgURE 2. Hook effect evaluation of the lipoprotein-associated phospholipase A2 activity assay on the Dimension Vista analyser. Substrate depletion hooks back measured ODs only at recombinant lipoprotein-associated phospholipase A2 (Lp-PLA ${ }_{2}$ ) activities $>1000 \mathrm{U} / \mathrm{L}$. curve to determine the IC50 of the drug. Calculated in vitro IC50 of Darapladib was $<50 \mathrm{nM}$.

Open vial reagents stability on-board the Dimension Vista ${ }^{\oplus}$ was demonstrated for 28 days by mean $\%$ difference from initial values of $2.1 \%$ (range: $0.5 \%$ to $3.8 \%$ ) and $0.4 \%$ (range: $-3.1 \%$ to $2.7 \%$ ) for low and high activity controls, respectively.

Table 3 shows the main biochemical characteristics of 250 Italian adult healthy volunteers. In this population, Lp-PLA 2 activity levels presented a Gaussian distribution $(P=0.98)$ ranging from 71 to $290 \mathrm{U} / \mathrm{L}$, with an average activity of $182 \pm 44 \mathrm{U} / \mathrm{L}$

TABLE 3. Main clinical and laboratory characteristics of the study cohort of healthy donors

$\begin{array}{cc}\text { Males, } \mathbf{N} & 123 \\ \text { Females, N } & 127 \\ \text { Age, years } & 37(18-70) \\ \text { Glucose, } \mathbf{~ m m o l} / \mathbf{L} & 4.7 \pm 0.5 \\ \text { Triglycerides, } \mathbf{~ m m o l} / \mathbf{L} & 0.9 \pm 0.3 \\ \text { Total cholesterol, mmol/L } & 4.3 \pm 0.6 \\ \text { LDL, } \mathbf{~ m m o l} / \mathbf{L} & 2.8 \pm 0.5 \\ \text { HDL, } \mathbf{~ m m o l} / \mathbf{L} & 1.4 \pm 0.3\end{array}$

Age is presented as median (min-max). Results are presented as mean $\pm \mathrm{SD}$. 
TABLE 4. Lipoprotein-associated phospholipase A2 activity values in adult healthy subjects

\begin{tabular}{cccc}
\hline \multirow{2}{*}{ Percentile } & \multicolumn{3}{c}{ Lp-PLA activity, U/L } \\
\cline { 2 - 4 } & $\begin{array}{c}\text { Entire cohort } \\
(\mathrm{N}=250)\end{array}$ & $\begin{array}{c}\text { Males } \\
(\mathrm{N}=123)\end{array}$ & $\begin{array}{c}\text { Females } \\
(\mathrm{N}=127)\end{array}$ \\
\hline Minimum & 71 & 87 & 71 \\
$\mathbf{2 . 5}$ & 93 & 107 & 84 \\
$\mathbf{5}$ & 106 & 135 & 97 \\
$\mathbf{2 0}$ & 144 & 171 & 128 \\
$\mathbf{3 3}$ & 164 & 187 & 145 \\
$\mathbf{5 0}$ & 183 & 205 & 165 \\
$\mathbf{6 7}$ & 203 & 224 & 179 \\
$\mathbf{8 0}$ & 220 & 241 & 192 \\
$\mathbf{9 5}$ & 254 & 260 & 217 \\
$\mathbf{9 7 . 5}$ & 262 & 265 & 225 \\
Maximum & 290 & 290 & 262 \\
Mean & 182 & 203 & 161 \\
SD & 44 & 40 & 37 \\
\hline $\mathbf{2 . 5 - 9 7 . 5}$ & $93-262$ & $107-265$ & $84-225$ \\
\hline
\end{tabular}

Lp-PLA 2 - lipoprotein-associated phospholipase A2.

(mean $\pm \mathrm{SD}$ ). When separate statistical analysis was performed by gender, a Gaussian distribution was also observed for each gender (males, $\mathrm{P}=$ 0.90; females, $P=0.44$ ). Mean Lp-PLA 2 activity levels in males were significantly higher than in females (203 \pm 40 vs. $161 \pm 37 \mathrm{U} / \mathrm{L}$, respectively; $\mathrm{P}<$ 0.001). Lp-PLA 2 activity distribution amongst population percentiles and reference intervals are summarized in Table 4. In our population, no statistically significant correlation was found between Lp-PLA $A_{2}$ activity and LDL-cholesterol (directly measured), HDL-cholesterol, and triglycerides. A moderate positive association was observed between serum Lp-PLA 2 activity and total cholesterol $(r=0.29, P<0.001)$. No statistically significant correlation between Lp-PLA 2 activity and age.

\section{Discussion}

In consideration of the role of Lp-PLA 2 as a potential marker of vascular inflammation in atherosclerosis, we evaluated the analytical performance of the $\mathrm{PLAC}^{\oplus}$ Test for the measurement of $L \mathrm{p}-\mathrm{PLA}_{2}$ activity on the Siemens Dimension Vista 1500 platform, for a possible application in routine clinical chemistry. Performance and practicability of the Dimension Vista 1500 system are highly suitable for both routine and emergency use highthroughput system. Correlation studies were performed comparing our results obtained using the PLAC $^{\bullet}$ Test for Lp-PLA 2 Activity Kit on a Siemens Dimension Vista 1500 instrument to values previously established by the manufacturer using a Beckman Coulter AU400 platform. We show that the PLAC ${ }^{\oplus}$ Test exhibits very good overall analytical performance characteristics on the Dimension Vista ${ }^{\circ} 1500$, as revealed by the results obtained for intra- and inter-assay precision, accuracy, linearity and sensitivity. Our results are comparable to the performance characteristics stated in the package insert of the $\mathrm{PLAC}^{\oplus}$ Test for Lp-PLA, Activity Kit and to the assay performance established on other platforms and recently published by two independent groups $(18,19)$.

Substrate depletion experiments demonstrated that the $\mathrm{PLAC}^{\circ}$ Test can be used on a Dimension Vista ${ }^{\circ}$ analyzer to measure without analytical errors Lp-PLA 2 activities as high as $1000 \mathrm{U} / \mathrm{L}$. This particular feature would allow the accurate evaluation of patients at high and very high cardiovascular risk, whose Lp-PLA 2 activities could be more elevated. Analytical interference that may commonly be exerted by high concentrations of some endogenous substances (i.e., albumin, haemoglobin, and lipids) was also evaluated and turned out to be very moderate. Moreover, the Dimension Vista analyzer specifically alerted for potential interference according to standard HIL (haemolysis, icterus, and lipemia) indices. Open-vial stability of the PLAC Test ${ }^{\oplus}$ for Lp-PLA 2 Activity reagents stored on-board the Dimension Vista ${ }^{\oplus}$ was evaluated by means of repeated measurements of Lp-PLA 2 activity using the same reagent set, controls, and calibration curve. Reagents were stable for up to 4 weeks in the refrigerated compartment $\left(2-8^{\circ} \mathrm{C}\right)$ of the instrument, which is comparable to the stability claims reported by the manufacturer for the PLAC Test ${ }^{\oplus}$ for Lp-PLA 2 activity on other automated analytical systems. The maximum number of 
determinations performed with a single reagent cartridge (104 tests) is consistent with many other routine tests already validated for this analyzer.

Recently, Lp-PLA 2 has been considered as a possible target for the treatment of atherosclerosis, and the selective $\mathrm{Lp}-\mathrm{PLA}_{2}$ inhibitor Darapladib has been tested as a possible strategy for the prevention and treatment of cardiovascular disease (24). However, the clinical trials conducted so far only yielded unsatisfactory results, so there is no evidence yet to support a strategy of targeted Lp$\mathrm{PLA}_{2}$ inhibition $(25,26)$. We used Darapladib to evaluate inhibitor effect on Lp-PLA 2 activity in our samples. In agreement with previous findings, our selective inhibition results confirmed that the $\mathrm{PLAC}^{\circledast}$ Test allows the specific measurement of Lp$\mathrm{PLA}_{2}$ activity (24).

In the present work we also showed the results of preliminary population studies performed on a cohort of 250 Italian apparently healthy volunteers (123 males, 127 females, aged 18-70 years). We found that Lp-PLA 2 activities presented a Gaussian distribution in the whole population as well as in the two gender groups, with mean Lp-PLA 2 activity in males significantly higher than in females $(P<$ 0.001 ) which is in agreement with previously published studies $(27,28)$. Moreover, this study establishes for the first time RIs of serum LP-PLA 2 activity in a healthy Italian population and proposes gender-specific Rls (107 - 265 U/L for males and 84 - $225 \mathrm{U} / \mathrm{L}$ for females) for clinical practice.

Recent recommendations have been issued for Lp-PLA $A_{2}$ activity measurement especially in intermediate cardiovascular risk patients, in order to decide whether they should be moved to a higher risk category $(16,17)$. However, uniform reporting of clinically relevant cut-points for Lp-PLA 2 activity in terms of increased risk for cardiovascular events has not been achieved yet. As claimed by the manufacturer, the $\mathrm{PLAC}^{\oplus}$ Test incorporates a cut point of $225 \mathrm{U} / \mathrm{L}$ for Lp-PLA $\mathrm{A}_{2}$ activity (which is expressed as $\mathrm{nmol} / \mathrm{min} / \mathrm{mL}$ in the package insert), which would identify patients at increased risk for coronary heart disease (CHD) events. This value was derived from the results obtained by the analysis of plasma samples from 5446 subjects in the placebo arm of the JUPITER Study, by which a cut point of $225 \mathrm{U} / \mathrm{L}$ was derived to differentiate subjects at increased risk for CVD events by Cox proportional hazards regression (29). Use of the proposed cut-point in our whole study population identifies $84 \%$ and $16 \%$ of the 250 subjects with low or high activity, respectively. In a population outcome-based sub-study conducted on nearly 5000 subjects from the REGARDS Study, it has been shown that Lp-PLA 2 activity above the sexspecific $80^{\text {th }}$ percentile $(250 \mathrm{U} / \mathrm{L}$ in males, $200 \mathrm{U} / \mathrm{L}$ in females) was associated with CHD risk over 5.3 years by Cox proportional hazards regression (30). These values are very similar to the sex-specific $80^{\text {th }}$ percentiles of our study population ( $241 \mathrm{U} / \mathrm{L}$ in males, $192 \mathrm{U} / \mathrm{L}$ in females).

In the healthy donor study cohort, there was no statistically significant association between age and Lp-PLA 2 activity, while a moderate positive correlation was observed with Lp-PLA $\mathrm{A}_{2}$ activity and total cholesterol, but not LDL cholesterol. This may well be due to the fact that part of $L p-P L A_{2}$ is carried by HDL also, while total cholesterol, which reflects the sum of $L D L, H D L$ and, to a lower extent, other cholesterol carrying lipoproteins, is more directly associated with circulating LP-PLA .

Although our research has reached its aims, this study has some limitations. In particular, the selected population of healthy donors is relatively young (median age 37 years), and therefore our findings may not translate to older populations. Nevertheless, our preliminary data are encouraging, and we are planning to extend this study in the future by increasing the number and age of enrolled participants. We also believe that further analysis of potential endogenous interferents (for example, higher concentrations of triglycerides) as well as selected exogenous agents such as lipid lowering drugs, would be valuable in order to meet suitable analytical conditions for the measurement of Lp-PLA 2 activity in patients with treated and untreated dyslipidemia.

Based on our results, we can conclude that the PLAC $^{\circledR}$ Test allows accurate and precise measurement of serum Lp-PLA 2 activity on the Dimension Vista $^{\circ} 1500$ automatic analyzer. Our findings add to the results previously obtained on other automatic clinical chemistry analyzers, and can con- 
tribute to the method harmonization process for Lp-PLA 2 activity measurement $(18,19)$. Taken together, the high-throughput potential of the Dimension Vista 1500 analyzer and the overall performance characteristics of the PLAC ${ }^{\circ}$ Test could provide a valuable tool for cardiovascular risk assessment in both primary and secondary prevention of cardiovascular disease, as well as for epidemiological investigations.

\section{References}

1. Farr RS, Cox CP, Wardlow ML, Jorgensen R. Preliminary studies of an acid-labile factor (ALF) in human sera that inactivates platelet activating factor (PAF). Clin Immunol Immunopathol 1980;15:318-30. https://doi.org/10.1016/00901229(80)90044-6

2. Tew DG, Southan C, Rice SQ, Lawrence MP, Li H, Boyd HF, et al. Purification, properties, sequencing, and cloning of a lipoprotein-associated, serine-dependent phospholipase involved in the oxidative modification of low-density lipoproteins. Arterioscler Thromb Vasc Biol 1996;16:591-9. https:// doi.org/10.1161/01.ATV.16.4.591

3. Silva IT, Mello AP, Damasceno NR. Antioxidant and inflammatory aspects of lipoprotein-associated phospholipase $A_{2}\left(L p-P L A_{2}\right)$ : a review. Lipids Health Dis 2011;10:170. https://doi.org/10.1186/1476-511X-10-170

4. MacPhee $C H$, Moores KE, Boyd HK, Dhanak D, Ife RJ, Leach $C A$, et al. Lipoprotein-associated phospholipase A2, platelet activating factor acetylhydrolase, generates two bioactive products during the oxidation of low density lipoprotein: use of a novel inhibitor. Biochem J 1999;338:479-87. https:// doi.org/10.1042/bj3380479

5. Di Pietro N, Formoso G, Pandolfi A. Physiology and pathophysiology of oxLDL uptake by vascular wall cells in atherosclerosis. Vascul Pharmacol 2016;84:1-7. https://doi. org/10.1016/j.vph.2016.05.013

6. Gonçalves I, Edsfeldt A, Ko NY, Grufman H, Berg K, Björkbac$\mathrm{ka} \mathrm{H}$, et al. Evidence supporting a key role of Lp-PLA2-generated lysophosphatidylcholine in human atherosclerotic plaque inflammation. Arterioscler Thromb Vasc Biol 2012;32:1505-12. https://doi.org/10.1161/ATVBAHA.112.249854

7. Packard CJ, O'Reilly DS, Caslake MJ, McMahon AD, Ford I, Cooney J, et al. Lipoprotein-associated phospholipase A2 as an independent predictor of coronary heart disease. West of Scotland Coronary Prevention Study Group. N Engl J Med 2000;343:1148-55. https://doi.org/10.1056/ NEJM200010193431603

8. Zalewski A, Macphee C. Role of lipoprotein-associated phospholipase A2 in atherosclerosis: biology, epidemiology, and possible therapeutic target. Arterioscler Thromb Vasc Biol 2005;25:923-31. https://doi.org/10.1161/01. ATV.0000160551.21962.a7

\section{Acknowledgments}

We would like to thank diaDexus, Inc. for providing $L p-P L A_{2}$ activity assay reagents.

\section{Potential conflict of interest}

None declared.

9. Oei $H H$, van der Meer IM, Hofman A, Koudstaal PJ, Stijnen $T$, Breteler $M M$, et al. Lipoprotein-associated phospholipase A2 activity is associated with risk of coronary heart disease and ischemic stroke: the Rotterdam Study. Circulation 2005;111:570-5. https://doi.org/10.1161/01. CIR.0000154553.12214.CD

10. Kolodgie FD, Burke AP, Skorija KS, Ladich E, Kutys R, Makuria $A T$, et al. Lipoprotein-associated phospholipase A2 protein expression in the natural progression of human coronary atherosclerosis Arterioscler Thromb Vasc Biol 2006;26:252329. https://doi.org/10.1161/01.ATV.0000244681.72738.bc

11. Younus A, Humayun C, Ahmad R, Ogunmoroti O, KandimaIla Y, Aziz M, et al. Lipoprotein-associated phospholipase A2 and its relationship with markers of subclinical cardiovascular disease: A systematic review. J Clin Lipidol 2017;11:32837. https://doi.org/10.1016/j.jacl.2017.02.005

12. Koenig W, Khuseyinova N, Löwel H, Trischler G, Meisinger C. Lipoprotein-associated phospholipase A2 adds to risk prediction of incident coronary events by C-reactive protein in apparently healthy middle aged men from the general population: results from the 14-year follow-up of a large cohort from southern Germany. Circulation 2004;110:1903-8. https://doi.org/10.1161/01.CIR.0000143377.53389.C8

13. Thompson A, Gao P, Orfei L, Watson S, Di Angelantonio E, Kaptoge $S$, et al. Lp-PLA(2) Studies Collaboration. Lipoprotein-associated phospholipase A2 and risk of coronary disease, stroke, and mortality: collaborative analysis of 32 prospective studies. Lancet 2010;375:1536-44. https://doi. org/10.1016/S0140-6736(10)60319-4

14. Ridker PM, Rifai N, Rose L, Buring JE, Cook NR. Comparison of $C$-reactive protein and low-density lipoprotein cholesterol levels in the prediction of first cardiovascular events. $N$ Engl J Med 2002;347:1557-65. https://doi.org/10.1056/NEJMoa021993

15. Daniels LB, Laughlin GA, Sarno MJ, Bettencourt R, Wolfert $R L$, Barrett-Connor E. Lipoprotein-associated phospholipase A2 is an independent predictor of incident coronary heart disease in an apparently healthy older population: The Rancho Bernardo Study. J Am Coll Cardiol. 2008;51:913-9. https://doi.org/10.1016/j.jacc.2007.10.048 
16. Davidson MH, Corson MA, Alberts MJ, Anderson JL, Gorelick $P B$, Jones $P H$, et al. Consensus panel recommendation for incorporating lipoprotein-associated phospholipase A2 testing into cardiovascular disease risk assessment guidelines. Am J Cardiol 2008;101:51F-7F. https://doi.org/10.1016/j. amjcard.2008.04.019

17. Perk J, De Backer G, Gohlke H, Graham I, Reiner Z, Verschuren $M$, et al. European Guidelines on cardiovascular disease prevention in clinical practice (version 2012). The Fifth Joint Task Force of the European Society of Cardiology and Other Societies on Cardiovascular Disease Prevention in Clinical Practice (constituted by representatives of nine societies and by invited experts). Eur Heart J 2012;33:1635-701. https://doi.org/10.1093/eurheartj/ehs092

18. Donato LJ, Meeusen JW, Callanan H, Saenger AK, Jaffe AS. Advantages of the lipoprotein-associated phospholipase A2 activity assay. Clin Biochem 2016;49:172-5. https://doi. org/10.1016/j.clinbiochem.2015.09.002

19. Cerelli MJ, Grimm K, Duan X, Mulberg E, Jalilie M, Sekella P, et al. Evaluation of recombinant enzyme calibration to harmonize lipoprotein-associated phospholipase A2 activity results between instruments. Clin Biochem 2016;49:480-5. https://doi.org/10.1016/j.clinbiochem.2015.11.018

20. McConnell JP, Hoefner DM. Lipoprotein-associated phospholipase A2. Clin Lab Med 2006;26:679-97. https://doi. org/10.1016/j.cll.2006.06.003

21. Kosaka T, Yamaguchi M, Soda Y, Kishimoto T, Tago A, Toyosato $M$, et al. Spectrophotometric assay for serum platelet-activating factor acetylhydrolase activity. Clin Chim Acta 2000;296:151-61. https://doi.org/10.1016/S00098981(00)00216-3

22. Clinical and Laboratory Standards Institute. User verification of performance for precision and trueness; approved guideline - 2nd ed. CLSI document: EP15-A2. Wayne, PA, USA: CLSI, 2005.

23. Clinical Laboratory Standards Institute. Defining, establishing and verifying reference intervals in the clinical laboratory; approved guideline - 3rd ed. CLSI document: C28-A3. Wayne, PA, USA: CLSI, 2010.
24. Blackie JA, Bloomer JC, Brown MJ, Cheng HY, Hammond $B$, Hickey $D M$, et al. The identification of clinical candidate SB-480848: a potent inhibitor of lipoprotein-associated phospholipase A2. Bioorg Med Chem Lett 2003;13:1067-70. https://doi.org/10.1016/S0960-894X(03)00058-1

25. White HD, Held C, Stewart R, Tarka E, Brown R, Davies RY, et al. Darapladib for Preventing Ischemic Events in Stable Coronary Heart Disease. The N Engl J Med 2014;370:1702-11. https://doi.org/10.1056/NEJMoa1315878

26. O'Donoghue ML, Braunwald E, White HD, Lukas MA, Tarka E, Steg PG, et al. Effect of darapladib on major coronary events after an acute coronary syndrome: The SOLID-TIMI 52 randomized clinical trial. JAMA 2014;312:1006-15. https://doi.org/10.1001/jama.2014.11061

27. Brilakis ES, Khera A, McGuire DK, See R, Banerjee S, Murphy $S A$, et al. Influence of Race and Sex on Lipoprotein-Associated Phospholipase A2 Levels: Observations from the Dallas Heart Study. Atherosclerosis 2008;199:110-5. https://doi. org/10.1016/j.atherosclerosis.2007.10.010

28. Garg PK, McClelland RL, Jenny NS, Criqui MH, Greenland $P_{1}$ Rosenson RS, et al. Lipoprotein-associated phospholipase A2 and risk of incident cardiovascular disease in a multi-ethnic cohort: The multi ethnic study of atherosclerosis. Atherosclerosis 2015;241:176-82. https://doi.org/10.1016/j. atherosclerosis.2015.05.006

29. Ridker PM, MacFadyen JG, Wolfert RL, Koenig K. Relationship of lipoprotein associated phospholipase A(2) mass and activity with incident vascular events among primary prevention patients allocated to placebo or to statin therapy: an analysis from the JUPITER trial. Clin Chem 2012;58:877-86. https://doi.org/10.1373/clinchem.2011.180281

30. Cushman $M$, Judd S, Kissela B, Jenny $N$, Elkind $M$, Safford M. Lipoprotein associated phospholipase A2 (Lp-PLA2) activity and coronary heart disease risk in a biracial cohort: The reasons for geographic and racial differences in stroke (REGARDS) cohort. Atherosclerosis 2015;241:e9. https://doi. org/10.1016/j.atherosclerosis.2015.04.048 